\title{
ERRATUM
}

\section{Erratum to: Electrochemical removal of nitrate in industrial wastewater}

\author{
Dong Xu, Yang Li, Lifeng Yin, Yangyuan Ji, Junfeng Niu, Yanxin Yu (ه) \\ (C) Higher Education Press and Springer-Verlag GmbH Germany, part of Springer Nature 2018
}

Redaction to: Front. Environ. Sci. Eng. 2018, 12(1): 9

https://doi.org/10.1007/s11783-018-1033-z

The original version of this article on Page 8, Section 4.1 stated that

"Electrodialysis is a process that uses selectively permeable membranes to segregate charged species. Membranes that are permeable to either cations or anions can be modified by affixing either negatively or positively charged groups to a polymer substrate. When cation and anion permeable membranes are alternately stacked and separated by water-filled spacers, with an electrode at either end of the stack, a voltage difference between the electrodes will cause cations to move toward the cathode and anions to move toward the anode, in the case of nitrated wastewater, the electrolysis is usually carried out with a nitrate selective membrane (Fig. 3) [106]. Electrolysis reactions at the two electrodes produce gases, primarily oxygen and hydrogen, which are carried off in a recirculating stream and released to the atmosphere" should be replaced by

"Baker and Griffis [106] presented a clear description on the mechanisms of electrodialysis. As described by Baker and Griffis [106], electrodialysis is a process that uses selectively permeable membranes to segregate charged species. Membranes that are permeable to either cations or anions can be modified by affixing either negatively or positively charged groups to a polymer substrate. When cation and anion permeable membranes are alternately stacked and separated by water-filled spacers, with an electrode at either end of the stack, a voltage difference between the electrodes will cause cations to move toward the cathode and anions to move toward the anode, in the case of nitrated wastewater, the electrolysis is usually carried out with a nitrate selective membrane (Fig. 3) [106]. Electrolysis reactions at the two electrodes produce gases, primarily oxygen and hydrogen, which are carried off in a recirculating stream and released to the atmosphere, as illustrated in detail by Baker and Griffis [106]".

The authors do apologize to John M. Baker and Timothy J. Griffis for their inappropriate expression on the citation and not giving them full credit for their verbatim use of the discussion about this topic.

The online version of the original article can be found at https://doi.org/10.1007/s11783-018-1033-z 